\title{
Effective Approaches to Enlarge English Vocabulary
}

\author{
Zhou Haixia $^{1}$, Yang Zhaohui ${ }^{2}$ \\ ${ }^{1}$ Department of International Changes, Hebei Institute of Communications, Shijiazhuang, China \\ ${ }^{2}$ Department of Scientific and Research, Hebei College of Industry and Technology, Shijiazhuang, China
}

Email address:

yangzhaohui2006@aliyun.com (Yang Zhaohui)

\section{To cite this article:}

Zhou Haixia, Yang Zhaohui. Effective Approaches to Enlarge English Vocabulary. International Journal of Language and Linguistics. Vol. 3, No. 6, 2015, pp. 394-400. doi: 10.11648/j.ij11.20150306.22

\begin{abstract}
This thesis is the study of enlarging English vocabulary based on the principles of paying attention to culture differences, making full use of context, enlarging English vocabulary through collocation and connotation. This thesis is based on some theories provided by the scholars of the field of English vocabulary learning. According to Harmer (2002:153), "if language structure makes up the skeleton of language, then it is vocabulary that provides the vital organs and the flesh. An ability to manipulate grammatical structure does not have any potential meaning unless words are used." "Culture consists of all the shared products of human society (Robertson, 1981)." It means language is a part of culture. Also, It is accepted that choosing words carefully in certain situations is more important than choosing grammatical structures (Harmer 1991). As words often appear in the form of collocation, English learners should enlarge their vocabulary not only with their meanings but also their collocations. This thesis illustrates presentation approaches for enlarging vocabulary, effective approaches of consolidating vocabulary, developing vocabulary enlarging approaches. Through these approaches, teachers will help students save large amount of time and efforts and create a more relaxed and efficient English learning environment for better performance.
\end{abstract}

Keywords: Vocabulary, Necessary, Language Learning

\section{Introduction}

As is described in the new syllabus, the fundamental aim of the new curriculum is to develop students' comprehensive language competence. The new English curriculum strives to accomplish far more than just help students learn English. It is also clearly stated that learning English should involve helping students 1)develop a certain level of comprehensive language competence and the ability to use language for real communication; 2)master certain basic language knowledge; 3)master listening, speaking, reading and writing skills. To accomplish these tasks, teachers should spare no effort to help students find out some effective ways to take a good command of English.

During the process of teaching and learning English, vocabulary teaching and learning is a very important part. According to Harmer (2002:153), "if language structure makes up the skeleton of language, then it is vocabulary that provides the vital organs and the flesh. An ability to manipulate grammatical structure dose not have any potential meaning unless words are used." Wilkins (1978:111), a famous British linguist, also claims, "Grammar provides the overall patterns, and vocabulary is the material to put in the patterns. While without grammar, very little can be conveyed, without vocabulary nothing can be conveyed."

Vocabulary is central to language and important to the typical language learner. Some second language researchers' quotations illustrate this point as the following: "One can't speak, understand, read or write a foreign language without knowing a lot of words. Therefore, vocabulary is at the heart of mastering a foreign language". (Rubin \& Thompson, 2004, p. 79) "...vocabulary is necessary in every stage of language learning ". (Laufer, 1997, p.140) "Words are basic building blocks of language". (Brown, 2001, p365)

Only when students have built up enough vocabulary, can they have a good grasp of the four skills--- listening, speaking, reading, and writing. Thus, the study of English vocabulary is seen as a basis for language acquisition.

However, many scholars abroad have concentrated their studies on German, Spanish and Russian rather than on English, as a second language or foreign language. Moreover, all the languages mentioned above all belong to the same family and undoubtedly there are a lot in common; Chinese students have more difficulties in English learning because there are big differences between these two languages.

In recent years many experts and scholars in China have 
also done a lot of research on English learning strategies, but not enough on vocabulary strategies.

What here should be mentioned is that in the new National English Curriculum syllabus, 7-level students are supposed to have a vocabulary of 2400 to 2500 words and 400 to 500 phrases; 8-level students are required to grasp 3660 words upon graduation from high school; able students are made sure to have access to a vocabulary of 4500 words. Therefore it become more necessary come up with some effective and efficient ways o help students grasp the basic vocabulary and enlarge a larger vocabulary.

\section{Theoretical Bases}

\subsection{Principles for Enlarging English vocabulary}

Enlarging English vocabulary does not simply mean learning and remembering words and their meanings from a vocabulary list or an English dictionary. It has its principles to comply with.

\subsubsection{Paying Attention to Culture Differences Loaded by Vocabulary}

Culture refers to the total pattern of beliefs, customs, institutions, objects, and techniques that characterize the life of a human community. "Culture consists of all the shared products of human society (Robertson, 1981)." This means not only such material things as cites, organizations and schools, but also non-material things such as ideas, customs, family patterns, and languages. Putting it simply, culture refers to the entire way of life of society, "the ways of a people." Language is a part of culture, it is influenced and shaped by culture, as culture is the symbolic representation of a people, and comprises their historical and cultural backgrounds as well as their approaches to life and their ways of living and thinking. Therefore, in the enlarging of English vocabulary, students must always be ready to get familiar with the cultural information loaded by words so that they can use the words appropriately and the obstacles caused by cultural differences will be eliminated. In a certain sense, culture helps to determine meaning. Students must always be aware of the importance of culture provision. The following examples may illustrate this point.

"Red", in China, is a color that can make people feel happy and excited; while in American history there once appeared "red scare".

"Individualism" in China always means somebody is selfish, or self-centered. It is a complete derogatory word; while in English spoken countries, it means independence, self-reliance or originality.

Therefore, learning about a word's related culture greatly contributes to students' mastering the word including the emotion and attitude it expresses.

\subsubsection{Making Full Use of Context}

Context refers to the phrase or even a longer utterance or a text that occurs before and/or after a word or phrase. The same word placed in different contexts has different meanings.
It often helps in understanding the particular meaning of the word, phrase, etc. For example, the word loud in loud music is usually understood as meaning "noisy" whereas in a tie with loud pattern it is understood as "unpleasantly colorful". Another example: I'll buy you a present when I come back. The word "buy" in that sentence means what it usually means. When it occurs in this sentence, "I won't buy it", it means "believe". Therefore, learning and enlarging their vocabulary, students should take into full consideration its context.

\subsubsection{Enlarging English Vocabulary Through Collocation}

It is accepted that choosing words carefully in certain situations is more important than choosing grammatical structures (Harmer 1991). As words often appear in the form of collocation, English learners should enlarge their vocabulary not only with their meanings but also their collocations. For example, when students learn a word like "take", it is much easier for them to master it knowing such collocations as take off, take up, and take away, and so on. Knowing the meanings and usages of those collocations, they have learned a large part of the word. Therefore, English learners should try to enlarge their vocabulary through their regular collocations.

\subsubsection{Enlarging English Vocabulary Through Connotation}

During the process of learning vocabulary, English learners will notice that the meanings of a word a dictionary provides do not apply to all situations. Meaning varies according to the expressions and phrases the word is placed in and the user's feelings and situations he/she stands in. Thus, a word has its connotative meanings except the denotative meanings.

For example, "Tom's face is quite familiar to Jack." It means Jack recognizes tom once he sees tom. Another sentence "Jack is too far familiar to tom's wife". When tom hears that, he must fly into rage because "familiar" in this sentence is a commendatory term which means there's a special relationship between Jack and tom's wife.

Another example, two words "escape" and "flee", both mean someone running away when facing something dangerous or urgent, but a slight difference between them is that "flee" more emphasizes scuttling away under emergent situations.

Thus, English learners enlarging their vocabulary should try to learn its connotations so that they can distinguish synonyms and learn about the feelings and attitudes behind the words.

\subsection{Definition of Vocabulary}

According to the Longman Dictionary of Language Teaching \& Applied Linguistics, Vocabulary is a set of lexemes, including single words, compound words and idioms, which function as a single meaning unit, regardless of the number or orthographical words it contains. "Date", "pain-induced" and "bird's view" are all lexical items. That is, the lexicon stores semantic units as both single and multi-word items. For example, 'work' is an item, but is 'work day' one or two? 'work-day' has a single meaning which is different from that of 'work' and 'day', but it is 
made up of two words. How about 'out of question'?

In this thesis, whenever "vocabulary" is mentioned, it means a combination of above two arguments: It refers to both single words and phrases for English language and chunks used by native speakers because part of vocabulary is acquired in the form of single words, and part of it is learned and memorized in the form of chunks, such as How do you do, What do you think, As far as I know..., It's reported that... and so forth. By the way, Word is used mainly to refer to an individual written form or sound (or combination of sounds) with meaning(s) in this thesis. Lexical item is another term used to refer to a certain vocabulary.

\subsection{The Content of Knowing Vocabulary}

Most English learners begin their English learning by starting with vocabulary and the vocabulary learning seems to accompany them throughout the whole learning process. Thus, how to help them to learn vocabulary effectively and efficiently has become a fairly important task for both teachers and students A question here should be first raised concerning the teaching and learning of vocabulary is: What does it mean to know a word? It is evident that without a proper understanding of this question, people cannot be able to develop effective strategies for teaching and learning vocabulary.

Wang Qiang (2000:118) gives a simple answer to this question, 1) knowing a word means knowing its pronunciation and stress;2) knowing a word means knowing its spelling and grammatical properties; 3 ) knowing a word means knowing its meaning; 4) knowing a word means knowing how and when to use it to express the intended meaning.

Firstly, learning a word demands to learn more than just the word itself. A vocabulary item may consist of more than just one word as mentioned in the definition of vocabulary. Thus, it is more sensible to learn such cases as a lexical item (Ur, 1996). McCarthy, from the point of view of word-formation, claims that 'a word must consist of at least one potentially freestanding morpheme', for example, 'playing' itself is freestanding with two morphemes 'play' and '-ing'. 'Do' is another freestanding item, while '-ing' is a bound item which cannot be used alone. Thus, a word may be in the form of a single morpheme like 'drink, desk, do' or in the form of more than one morpheme with different bounds such as 'repay' with prefix 're-', 'employer' with suffix '-er' or even compound words with more than one root but only having one identity like 'air-conditioning'. What's more, there are multi-word units such as phrasal verbs like 'break down', 'take off', etc., compound words like 'greenhouse', 'babysitter' etc., as well as idioms and metaphors. For the components and sequence of these words are fixed, they should be considered as one form (McCarthy, 1990).

However, knowing simply the form of a word is far from enough as students who know the formation of words or compound words may sometimes not know the meaning of them in contexts. It would be more difficult for students even if all the words in the idiom may be already known. According to Hege (2000), vocabulary learning involves more than two aspects of meaning. The first aspect of vocabulary learning is produced from the angle of its denotative and connotative meaning and the second aspect is produced from an angle of the sense relations among words.

\subsubsection{Denotative Meaning}

A denotative meaning of a word or a lexical item refers to those words that we use things as regards real objects, such as a sign or a name, etc. in the physical world. The denotative meaning is always the primary one of a word and it may seem quite easy to learn. However, problems also exist sometimes because people may not always be able to find the equivalent concepts of two languages. For instance, in English 'niece' and 'nephew' represent one brothers' or sisters' daughters and sons; in Chinese it is sharply different about the names used to represent one's brother's children and of one's sisters. The same problems also exist in many other areas. When it occurs, new concepts will be added to the vocabulary.

\subsubsection{Connotative Meaning}

According to Hedge (2000:112) a connotative meaning of a word refers to 'the attitudes or emotions of a language user in choosing a word and the influence of these on the listener or reader's interpretation of the word'. These would include words that may indicate subtle feelings or express a positive or negative attitude towards some -thing. He also explains, 'connotative meaning derives from a mix of cultural, political, social and historical sources and learners will be aware of this phenomenon in their own language' but may not be so aware of this in the target language. Here is an example from Ur (1996). He points out that the word 'dog', whose denotative meaning is the animal itself, has a connotative meaning that represents friendship and loyalty. However, in different cultures it may have different connotative meanings.

\subsubsection{Collocations}

Collocations refer to words that co-occur with high frequency and have been accepted by people as fixed ways for the use of words. For instance, in English, the words 'look', 'see', and 'watch' are similar in meaning but are always used with different collocations as people will say 'look at the blackboard', 'see a movie' and 'watch TV'. Similarly, people say 'heavy rain/fog/snow', 'heavy smoker', 'heavy traffic' but never say 'heavy wind' or 'heavy accident'. It is commonly believed that learning word collocations is in fact more effective than simply learning a single word at one time.

\subsubsection{Synonyms, Antonyms and Hyponyms}

Synonyms refer to items that have the same or nearly the same meaning. For instance, "valuable", "priceless" and "invaluable" are synonyms. Antonyms refer to items that have opposite meanings. For instance, "decrease" is an opposite of "increase". Hyponyms refer to items which can be listed together under the same superordinate concept. For example, under the concept of subject, words such as 'math, politics, chemistry, geography' and 'philosophy' may be included. 


\subsubsection{Receptive and Productive Vocabulary}

During the process of teaching and learning vocabulary, it is of great necessity for both teachers and students to have the idea of a distinction between vocabulary and productive/active vocabulary. Receptive/passive vocabulary refers to those words that learners are able to recognize and understand in reading or listening materials but unable to use automatically in speaking or writing. Productive/active vocabulary refers to those words that learners are able to recognize and understand in reading or listening materials but also able to use automatically in speaking and writing. At beginner's level, many new words learned by students generally have immediate practical use. therefore they can quickly be included in their active vocabulary. However, language learners, as they learn more and more new words, will find the fact that for some words, they are able to recognize and to use for speaking and writing; while for some words, they can simply able to recognize when met in reading. There are also many words that can gradually become students' active vocabulary after being met more and more times. Thus, Hedge (2000:116)proposes that students should regard the vocabulary knowledge as " a scale running from recognition of a word at one end to automatic production at the other, through immediate stages of making greater sense of the word and how it might be used in different contexts.' She also says that knowledge of some words will stay at the recognition end of the continuum and will never turn into part of students' productive vocabulary. Therefore, the teacher, when teaching vocabulary, should make clear what words should be included into the students' productive or active vocabulary so that they can organize proper activities to help students use these words automatically and passionately when they are speaking or writing.

Nation (2001: 26-28) explained about receptive and productive vocabulary in a more detailed way. According to him, receptive knowledge involves (1) being able to recognize the word when it is heard; (2) being familiar with its written form so that it can be immediately recognized when it is encountered in reading; (3) recognizing that it is made up of some parts and being able to relate these parts to its meaning; (4) knowing that the word signals a particular meaning; (5) knowing what the word means in the particular context in which it has just occurred; (6) knowing the concept behind the word which will allow understanding in a variety of contexts; (7) knowing that there are some related words; (8) being able to recognize that the word has been used correctly in the sentence in which it occurs; (9) being able to recognize the typical collocations; (10) knowing that the word is not an uncommon one and is not a pejorative word. Productive knowledge of a word includes receptive knowledge and extends it. It involves: (1) being able to say it with correct pronunciation including stress; (2) being able to write it with correct spelling; (3) being able to construct it using the right word parts in their appropriate forms; (4) being able to produce the word to express the meaning; (5) being able to produce the word in different contexts to express the range of meanings of it; (6) being able to produce synonyms and opposites for it; (7) being able to use the word correctly in an original sentence; (8) being able to produce words that commonly occur with it; (9) being able to decide to use or not use the word to suit the degree of formality of the situation.

\section{Effective Approaches in Enlarging English Vocabulary}

\subsection{Presentation Approaches for Enlarging Vocabulary}

There are a great variety of ways presenting vocabulary and different teachers may prefer to present vocabulary in their own different ways. For a more effective vocabulary teaching, it is always useful for a teacher to have a repertoire of techniques so that they can choose an appropriate way according to the type of vocabulary and different age level of the learners. The following suggestions may be of some help for a teacher.

1) The teacher may offer a visual or physical demonstration whenever possible, using cards, pictures, gestures or mime to show meaning.

2) The teacher may give a verbal context to show meaning and ask learners to give the meaning of it first before it is offered by the teacher.

3) The teacher can explain a word by using synonyms or antonyms.

4) The teacher can use hyponyms or lexical sets to show the relations of words and their meanings, e.g. body part: arm, leg, foot, hand.

5) The teacher may use the word-formation rules and common affixes to build new lexical knowledge on what has been already known.

6) The teacher may teach vocabulary

7) The teacher may teach vocabulary in chunks which refer to a group of words that go together to form meaning and co-occur with high frequency. According to lewis (2002:121), it refers to 'pre-fabricated formulaic items'.

8) The teacher should sometimes think about where the word may occur in real life and try to relate newly learned knowledge to students' life to stimulate their interest.

9) The teacher can try to provide different reading materials for introducing new words.

10) The teacher must be ready for any possible misunderstanding or confusion that students may meet.

\subsection{Effective Approaches of Consolidating Vocabulary}

For many foreign language learners, perhaps it is more difficult to consolidate and remember vocabulary items than to learn them for the first time. It is too often to be heard students complain that they keep learning and forgetting. This may be partially true. If students learn vocabulary $b$ 
themselves, it is very often rote learning whose effectiveness is seldom guaranteed. However, when they study vocabulary together or in groups, through different kind of activities and under the teachers' guidance, vocabulary learning will becomes more interesting and increases the effectiveness of consolidation. Furthermore, only very limited range of vocabulary exercises are provided in the textbooks for students, such as matching definitions, labeling and gaps filling, etc, while students always need more support and more varieties of exercises and activities to help them recycle and consolidate the newly learned vocabulary so that they can include them in active use.

\subsubsection{Labeling Pictures / Objects}

Show the students a picture and ask them to write the names of objects indicated in the picture. A competitive element may be added by making the first student to finish the winner.

\subsubsection{Spotting the Differences}

Put the students into pairs. Each member of the pair is given a picture which is slightly different from his partners. The pair hides the pictures from one another and then by a process of describing, questioning and answering, find out what the differences are.

\subsubsection{Describing and Drawing}

Put the students into pairs. One student gets a picture, the other a blank piece of paper and a pencil. The student must tell the other in English what to draw so that the drawing ends up the same as the original one. The student must not show the picture before the drawing is finished.

\subsubsection{Playing a Game}

The teacher shows the students a picture or a tray with many objects written in English on it, or a series of magazine pictures or flash cards. Give them two minutes to memorize as many of objects as they can. Then take the pictures or tray away and ask the students to say what they saw, or write down all they remember seeing, then compare with the rest of the class.

\subsubsection{Blackboard Bingo}

The teacher writes on the board 10 to 15 words that the students have recently learnt. Read the definition of 5 of them. The students should cross off all the 5 words that are defined. Whoever crosses off all of them first will shout 'Bingo' and he or she wins. This can be a competition among the students in a chain activity, which should be very motivating and beneficial to students' revision and memorization of words. These words may be nouns, verbs, adjectives and adverbs, etc.

\subsubsection{Synonyms and Antonyms}

Write on the board or dictate a series of 6 to 10 words that have synonyms and definite opposites. Divide the class into pairs. Ask them to give the synonyms and opposites of the words given. Note that there may be more than one opposite or synonym for one word, e.g. "hard"---“"soft", "gentle" or "easy".

\subsubsection{Grouping Words into Categories}

Ask the students to group words into different categories.

After teaching the topic of healthy and unhealthy food, teachers can ask the students to group the list of food and drink items into the appropriate categories as a way to help them.

Table 1. Appropriate categories.

\begin{tabular}{l|lll}
\hline pizza & candy & beans & ice cream \\
butter & hamburgers & garlic & cheese \\
pure water & cookies & pears & carrots \\
milk & green tea & coke & tomatoes \\
Good for one's health & Not so good for one's health \\
\hline
\end{tabular}

\subsubsection{Word Association}

The teacher writes a key word, e.g. family. And then ask the students to write down any word they can come up with connected with family in limited time, e.g. two minutes. When time's up, the student who writes down the highest number of acceptable words is the winner.

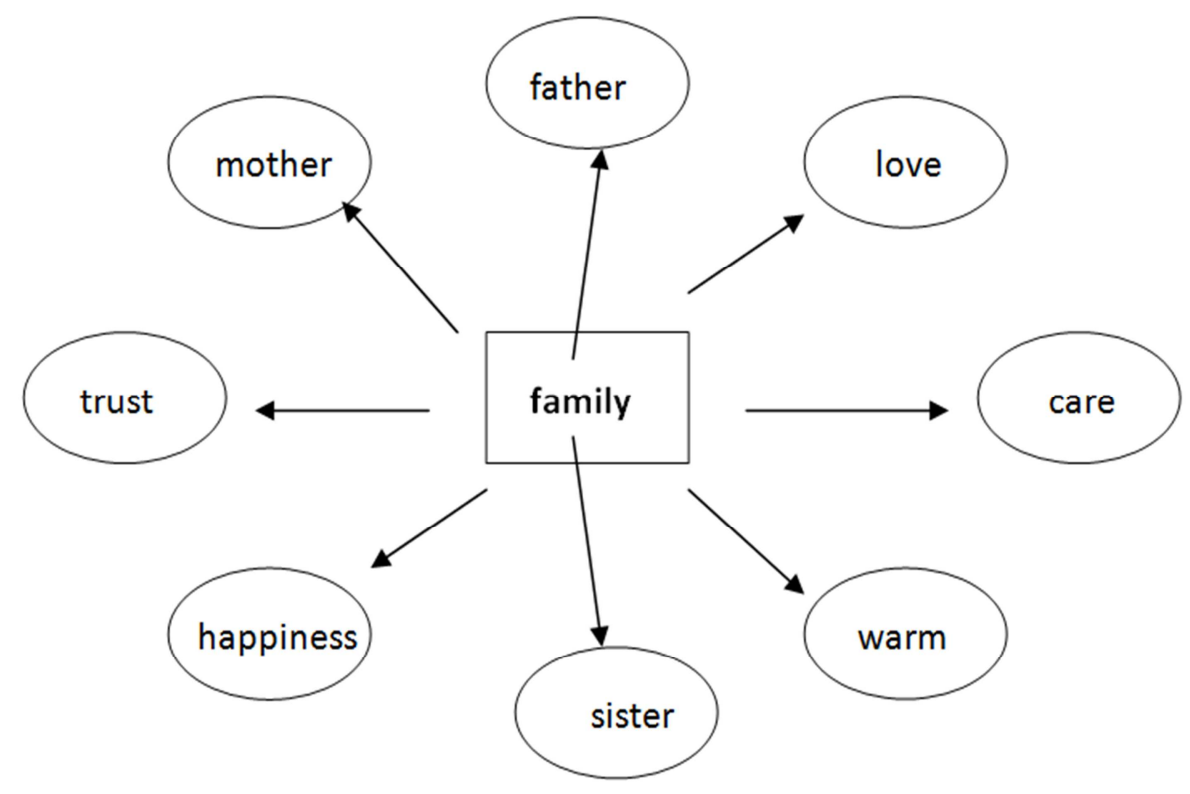

Figure 1. Word association. 


\subsubsection{Using the Internet Resources}

On the Internet there are many websites from which teachers can find more vocabulary activities that may be helpful to learners of different levels. Here is a website that may be useful.

http://www.teachingenglish.org.uk/try/vocabtry/vocab_acti vities.shtml\#bus

\subsection{Developing Vocabulary Enlarging Approaches}

As is known to all, students cannot be able to grasp all the English vocabulary simply from class teaching because of limited class time. Therefore, it is necessary for teachers to guide them to develop more vocabulary items learning strategies so that they can effectively acquire larger vocabulary by themselves out of class time. It demands the students themselves not only to keep regular review and consolidation for the old vocabulary, but also enrich themselves with new vocabulary through comprehensive reading.

\subsubsection{Regular Review}

No one can remember and grasp every new word until they have met with it at least five or seven times. Hermann Ebbinghaus, a German psychologist, do a more systematic research on forgetting phenomenon. According to his forgetting curve, it can save the learners $58.2 \%$ of time to remember the information material for the second time one hour after their first remembering; $33.7 \%$ one day after and $25 \%$ six days after. Memory strength gradually recedes with time and forgetting speed is always very fast at the beginning but gradually declines. The newly remembered words are always soon forgotten out. If they are reviewed soon after the student forgets, memory will be strengthened; while if they are reviewed after they are completely forgotten.

Thus, newly learnt words should be learned and remembered for several times. English learners may make full use of morning, evening and other available time to remember. Every time may not be long so as not to make the brain cortex tired and cause pressure which will in turn affect remembering Repeatedly short-time language contacts are more advantaged than long-time continuing remembering. Every week the students can review the newly learnt vocabulary in recent week. Every month do the same. At the time obtaining new words, they should also pay attention to reviewing old words. Timely regular review is the most basic method that can help students overcome with forgetting, comprehend and consolidate knowledge.

\subsubsection{Guessing Meaning from Context}

It is not a new idea to encourage English learners to guess the meaning of unfamiliar words or expressions through context, but the problem is how to develop this ability. At the beginning learners need teachers' guidance concerning what contextual clues to find and how they can contribute to the discovery of meaning. Usually, the topic, the grammatical structure, the possible meaning between the given words and other words and the linguistic pattern where the words appear, all these may give hints to the meaning in one way or another. Here is an example:

"Let's synchronize our watch so that we can start the game at the exact time." If students don't know the meaning of the word "synchronize", there are enough clues for them to guess.

\subsubsection{Organizing Vocabulary in Special Ways}

There is evidence that if information is organized and stored in special ways, e.g. related information is stored together or new information is related to previously stored information, it is more likely to be remembered and easier to retrieve. Considering the tremendous task to remember large vocabulary, it seems more necessary for English learners to organize their vocabulary in an effective way. For instance, teacher can ask them to keep a notebook. Instead of putting all the words they learned in a random way, they can organize the vocabulary according to different categories or topics. For example, words like library, student, teacher and textbook can be included under topic of school; fork, knife, menu, waiter under topic of restaurant.

\subsubsection{Using a Dictionary and Keeping a Note}

Using a dictionary properly is a very important of vocabulary learning, and students should be guided to use it at the beginning. Normally students should not be encouraged consult dictionaries for every word they do not know. The students should first try to guess its meaning from the context. If they still cannot get the meaning after it has been repeated for many times, a dictionary will be necessary. Learners need to be told that they should choose them according to their specific language context.

In a sense, a dictionary can be one compiled by students themselves, that is, their own vocabulary notebook. Schmitt and Schmitt (1995) argue that keeping vocabulary notebooks is one way of promoting earning independence. It is also believed that learners' lexical competence. Fowle (2002) finds that the introduction of vocabulary notebooks into the English language program in a secondary school can act as a tool to empower the learners to become more independent in their learning.

Although many students have a vocabulary notebook, not many of them can effectively compile their own vocabulary notebook. They may index their vocabulary either in an alphabetic order or topic group to arrange their vocabulary. It may include beautiful sentences, useful collocations, word families and new words they encountered in reading and listening materials, etc. In this way, the words they learnt can be easily found from their vocabulary book.

\subsubsection{Enlarging Vocabulary Through Comprehensive Reading}

Vocabulary knowledge is connected to reading comprehension. Extensive reading can increase vocabulary knowledge.

The incidental vocabulary learning hypothesis (Nagy \& Herman 1985:233-253) proposes that the vast majority of vocabulary words are learned gradually through repeated exposures in various discourse contexts. Proponent of this view state that learners typically need about ten or twelve exposures 
to a word over time in order to learn it well. Following this same logic, it is argued that English learners who achieve advanced reading proficiency in a language will acquire most of their vocabulary knowledge through extensive reading rather than through instruction. For example, Krashen (1989:440-464), a leading proponent of extensive reading, argues that language learners acquire vocabulary and spelling most efficiently by receiving comprehensible input while reading. He claims that the Input Hypothesis also applies to vocabulary acquisition by means of extensive reading.

Coady (1977:235) shows in his study that a great deal of foreign language vocabulary is indeed learned through extensive reading. However, it has also pointed out that we need to help beginners to learn a relatively small number of high frequent vocabulary words well so that they become automatic, sight vocabulary. Only with this fundamental competence will beginners be able to read independently and acquire the language in a natural order that is assured by so many pedagogical approaches today. In other words, instructional programs need to ensure that students know at least the basis vocabulary so that they can indeed vocabulary incidentally through extensive reading.

Yang Zhiqing \& Deng Wei (2001:68) suggest in their study that there are three stages of reading: the expending stage of vocabulary, the developing stage of reading skill, and the perfect stage of reading skills. During the first stage, students' vocabulary is not large enough for them to adequately use reading skills, preferably a direct approach to vocabulary teaching is adopted, rather than relying an extensive reading to expend their vocabulary.

In school, therefore, as students' vocabulary is not large enough, it is not always a good practice for instructors to emphasize that all students should expand their vocabulary through large amounts of extensive reading. It is not a good choice for one to use skills with limited vocabulary.

\section{Conclusion}

This thesis started with a definition of vocabulary and a question about what knowing a word involves. It is organized to show English learners the content of vocabulary learning before they try to think up ways to enlarge vocabulary.

It is a hard task to learn a word and take a good command of the vocabulary. Many teachers and students are aware of the importance of vocabulary in the process of foreign language learning and many learners devote large amounts of time to the learning and enlarging of vocabulary, but always receive dissatisfying outcomes. There are at least two reasons that can account for this. One is that students learn vocabulary incorrectly. They simply remember its Chinese translation in isolation of its context and don't know its connotative meaning, collocations or the formation rules. So when they are to accomplish a reading or listening task with those words used in different contexts or in combination with other words, they get confused easily with the meaning they remembered. Another one is that many students learn vocabulary in ineffective ways as they rote-learn all the vocabulary only before exams. After the exam, they quickly forget what they have learned. So it is very important to make students aware how to learn vocabulary effectively and how to use some vocabulary leaning strategies to help them learn.

The learning and enlarging of English vocabulary is a massive task that demands both teachers' effective instruction and learners themselves' efficient approaches. If teachers present enough vocabulary resources to obtain new vocabulary and help them review and consolidate old vocabulary in a scientific way, it will save students large amount of time and efforts and create a more relaxed and efficient English learning environment for better performance.

\section{References}

[1] Brown, H. D (2001). Teaching by Principles: an Introductive Approach to Language Pedagogy [M]. Beijing: Foreign Language Teaching and Research Press.

[2] Coady, J. 1997. L2 vocabulary acduisition through extensive reading [A]. In J. Coady \& T. Huckin9eds). Second Language vocabulary Acquisition [C] Cambridge University Press.

[3] Fowle, C. (2002) Vocabulary Notebooks: implementation and outcomes. ELT Journal 56/4:380-388.

[4] Harmer, J. 1991. The practice of English language teaching. Second Edition. London: Longman.

[5] Hedge, T. (2000) Teaching and Learning in the Language Classroom. Oxford: Oxford University Press.

[6] Krashen, 5.1989.We acquire vocabulary and spelling by reading: Additional evidence for the Input Hypothesis [J].Modern Language Journal 73:440-464.

[7] Laufer, B. (1997). "What's in a Word that Makes it Hard or Easy: Some Intralexical Factors that Affect the Learning of Words" [A]. In Schmitt, N \& McCarthy, M (eds.), Vocabulary: Description, Acquisition, Pedagogy [C]. Cambridge: CUP. p.140.

[8] Lewis. M. (2002:121) The Lexical Approach. Boston, MA. Heinle, Thomson.

[9] McCarthy, M. (1990). Vocabulary . Oxford: Oxford University Press.

[10] Nation, P. (2001). Learning Vocabulary in Another Language. Cambridge: Cambridge University Press.

[11] Nagy. W.E., Herman, P.A. \& Anderson, R.C. 1985. Learning words from context [J]. Reading Research Quarterly 20: 233-253.

[12] Schmitt N. \& Schmitt D. (1995). Vocabulary notebooks: theoretical underpinnings and practical suggestions. ELT Journal 49/2:133-43.

[13] Ur, P. (1996). A Course in Language teaching: Practice and Theory. Cambridge: Cambridge University Press.

[14] Wang Qiang (2000). A Course in English Language Teaching, Beijing: High Education Press.

[15] Wilkins, D. A. 1978. Linguistics in Language Teaching [M]. Edward Arnold. 